\title{
Epidemiology of Epstein-Barr virus in Malaysia
}

\author{
M. R. Norzuriza, W. Kon Ken, M. Mohammad, I. Isahak and M. M. Rahman ${ }^{7 *}$ \\ Department of Medical Microbiology and Immunology, Faculty of Medicine, University \\ Kebangsaan Malaysia, Cheras-56000, Kuala Lumpur, Malaysia
}

\begin{abstract}
An epidemiological investigation was carried out on patients reported to Hospital University Kebangsaan Malaysia (HUKM) suspected to be carriers of Epstein-Barr virus from July 2005 to June 2006. A total of 402 patients' sera were analyzed by Enzyme Immunoassay with Kit Enzygnost ${ }^{\circledR}$ by automated BEP ${ }^{\circledR} 2000$ instrument. Of these $91.3 \%$ were found to carry Epstein-Barr virus (EBV) antibodies. Among these, 90.4\% of male and $92.2 \%$ of female patients were positive: the difference was not significant. Considering community, $91.9 \%$ of Malay, $91.9 \%$ of Chinese, $75 \%$ of Indian and $93.3 \%$ of others were positive: the differences were not significant. Among the age groups, $77.5 \%$ of $0-20$ yearolds, $98.6 \%$ of $21-40$ year-olds, $96 \%$ of $41-60$ year-olds and $100 \%$ of those above 60 years of age were positive: these differences were significant $(\mathrm{p}<0.005)$. No significant difference in the prevalence of EBV antibodies existed between the months of sampling. In the present study, EBV-specific IgG and IgM antibodies were determined. The proportion of samples positive to IgM was much less than those positive to IgG. (Bangl. vet. 2008. Vol. 25 , No. 2, 82-87)
\end{abstract}

\section{Introduction}

Epstein-Barr virus (EBV) is a member of subfamily gammaherpesvirinae of the family herpesviridae. The virus can cause glandular fever, with swollen lymph nodes, sore throat, and fever. EBV is the most prolific viral contributor to the development of human lymphomas. In cases of immunodeficiency, such as AIDS, organ transplants, and genetic immune disorders, EBV can reactivate, causing proliferation of abnormal lymphocytes and the potential for several different lymphomas (Thompson and Kurzrock, 2004).

Recent evidence suggests that Hodgkin's lymphoma, T cell lymphomas, and some gastric carcinomas may be linked to EBV (Thompson and Kurzrock, 2004).

The detection of EBV-specific IgG plays a significant role in the monitoring of the immune status. In case of transplantation, the detection of EBV antibodies should lead to the exclusion of potential donors if the intended recipient tests negative for EBV antibodies.

The virus is spread mainly through saliva. It infects the squamous epithelial cells of the salivary gland, and B-lymphocytes. EBV induces the proliferation of

\footnotetext{
7 Department of Microbiology and Hygiene, Faculty of Veterinary Science, Bangladesh Agricultural University, Mymensingh, Bangladesh

*Correspondence: E-mail:- mostabau@yahoo.com
} 
infected B-cells. If the host immune system is unable to counteract this growth, that enhances the risk of cancer.

Immunosuppression through HIV can lead to an increase in latent EBV and subsequently to reactivation of disease (Rinaldo, 1990). Therefore, immunosuppression with herpes viruses is significant in patients with immuno-deficiencies (Anon, 2006a).

The present research was undertaken with cases of Hospital University Kebangsaan Malaysia (HUKM) from where a number of EBV suspected samples are sent to the Laboratory of the Department of Microbiology and Immunology for diagnosis. The serum samples were analyzed to assess Anti-EBV antibodies and the results were related to age, gender, community and month.

\section{Materials and Methods}

\section{Collection of samples}

A total of 402 serum samples from EBV-suspected patients were obtained by standard technique and sent in chilled condition to the Laboratory of the Department of Medical Microbiology and Immunology, UKM, Malaysia for diagnosis. The samples were from patients of different communities such as Malaya, Chinese, Indian and others and from different age groups: 0-20 years, 21-40, and 41-60 and above 60 years, and from male and female patients. The investigation was carried out round the year to observe the influence of different months on the prevalence of EBV infection.

\section{Diagnostic kits}

Kit Enzygnost Anti EBV/IgG and Enzygnost Anti EBV/IgM were obtained from Dade Behring Marburg GmbH, Emil-von-Behring-Str-76, D-35041, Marburg, Germany to perform Enzyme Immunoassay to determine specific IgG and IgM antibodies to EBV (Anon, 2006b). The antibodies bind to the antigen in the wall of the test plate. The anti-Human IgG/POD or IgM/POD conjugate binds to these specific antibodies. The enzyme component of the conjugate catalyzes the working chromogen solution, producing a blue colour. This reaction is terminated by the addition of the stopping solution POD with the colour changing to yellow. The intensity of the yellow colour is proportional to the activity of EBV-specific IgG/IgM antibodies in the sample.

Kits Enzygnost AntiEBV/IgG and AntiEBV /IgM contain Anti-EBV IgG test plate, Anti-EBV IgM test plate, Antihuman IgG/POD conjugate, Conjugate Buffer, Anti-EBV Reference P/N, Sample buffer, and Barcode.

\section{Protocol used}

Enzyme Immunoassay was performed to determine EBV-specific IgM and IgG using Kit Enzygnost Anti EBV/IgG and Enzygost Anti EBV/IgM. The test was 
performed using the BEP ${ }^{\circledR} 2000$. The instrument performed sample dispensing steps and subsequent processing steps automatically.

1. Internal quality control: A values obtained from the measurement of EBV antigens minus the value of the same sample obtained with the EBV control antigen was used in each case.

2. Validation criteria: The differences obtained per test plate with the anti-EBV Reference P/N (calculated from differences in A values for the measurements with EBV antigen and EBV control antigen) was within the upper and lower margin. In addition $A$ values did not differ by more than $\pm 20 \%$ from the mean values calculated from these values.

3. The calculations were performed automatically with $B E P^{\circledR} 2000$.

4. Correction factor: Mean value/Mean A value for reference P/N

5. Qualitative evaluation: The following criteria were followed for evaluation:

Anti-EBV /IgG or IgM negative A $<0.100$ (cut-off)

Anti-EBV/IgG or IgM positive A $>0.200$

\section{Statistical analysis}

SPSS package by Chi-square test was used to compare the prevalence of EBV infections in different categories.

\section{Results and Discussion}

A total of 402 serum samples were examined from July 2005 to June 2006. Of these, 91.3\% were positive for Anti-EBV IgG antibodies and $8.7 \%$ for Anti-IgM antibodies. Of the total, $90.4 \%$ of samples from male patients and $92.2 \%$ from female patients were positive for IgG (Fig. 1).

No significant difference in EBV infection was observed between male and female ( $p>0.001$ ). Regarding community, $91.9 \%$ of Malay, $91.9 \%$ of Chinese, $75 \%$ of Indian and $93.3 \%$ of others were positive for IgG (Fig. 2).

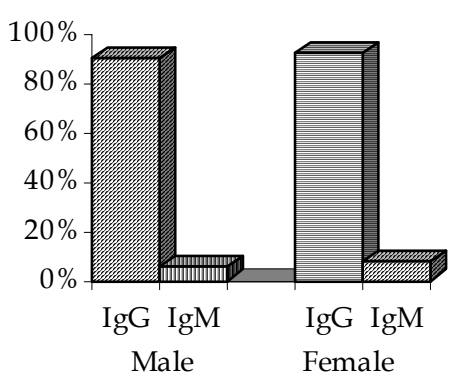

Fig. 1. EBV infections of male and female at HUKM reported cases in Malaysia

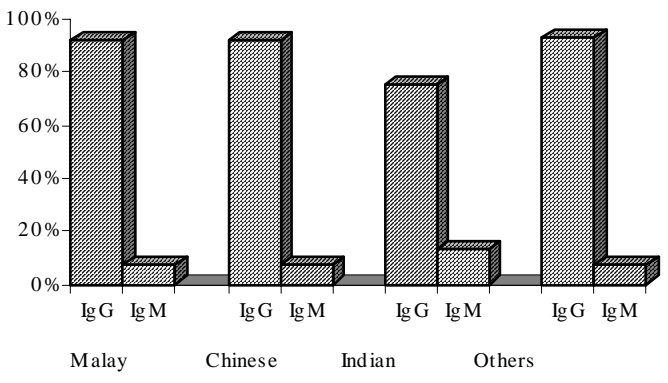

Fig. 2. Percentage of different communities whose serum was positive for Epstein-Barr virus antibodies 
No significant difference $(p>0.005)$ in prevalence existed between the communities. Studies in different age groups revealed that $77.5 \%$ of $0-20$ year- olds, $98.6 \%$ of $21-40$ year -olds, $96 \%$ of $41-60$ year-olds and $100 \%$ of those above 60 years were positive for IgG (Fig. 3).

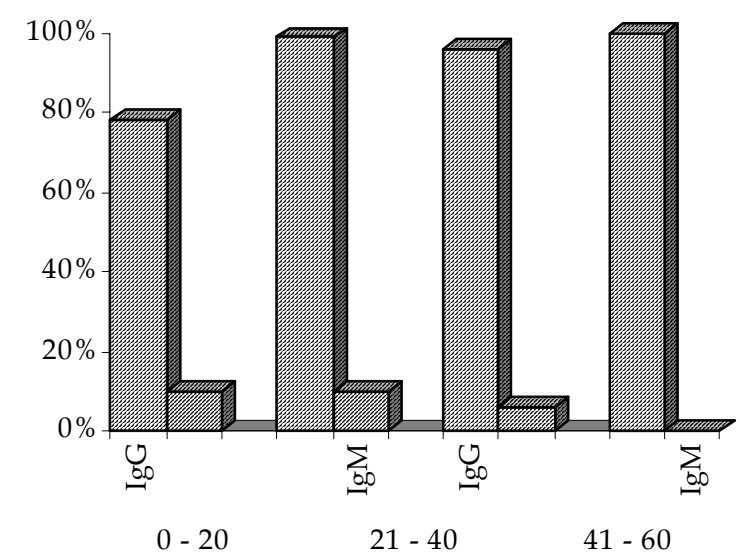

Fig. 3. EBV infection of different age groups at HUKM reported cases in Malaysia

The difference in prevalence between age groups was significant $(p>0.001)$. There was no significant difference in prevalence of EBV antibody in different months of sampling (Fig. 4).

It is evident from the present study that EBV infection is common in Malaysia. The percentage of infection increased with age: $77.5 \%$ of people between $0-20$ years were infected, and $100 \%$ of people aged more than 60 years. This indicates that almost all people in this area became infected with EBV during their lifetime. Latent EBV infection may lead to reactivation due to immuno-suppression through infection with HIV or lack of protective immunoglobulin (Anon, 2006a; Rinaldo, 1990).

Figure 4 EBV antibody pattern at HUKM reported cases July 2005 - June 2006 in Malaysia.

A similar study conducted by Haque et al. (2001) in Bangladesh showed a prevalence of EBV infection in the study group of $81.3 \%$. Only $42.4 \%$ of infants had antibodies to EBV by the age of 1 year, and a significant rise in the percentage of sero-positives was observed between 0-1- and 1-2-year-old children, indicating a high rate of primary infection at these ages. The authors mentioned that the prevalence of IgG antibody was $87.9 \%$ in the $2-10$ years age group and was sustained at over $85 \%$ thereafter.

Takeuchi et al. (2006) conducted a survey in Japan by seroprevalence during 1995-2006 and reported that most of the children were infected in their early life with EBV. They mentioned that $>90 \%$ of 5-9-year-old children were infected with EBV while the age of primary infection was delayed in Western countries where 
approximately $50 \%$ of $5-9$-year-old children were infected. They pointed out that later in life EBV-positive children could suffer from EBV-associated disorders.

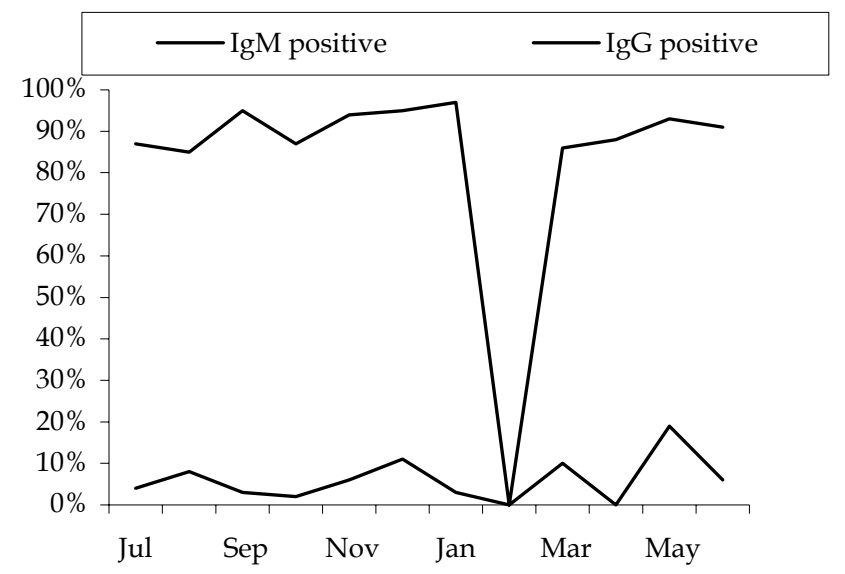

Fig. 4. EBV antibody pattern at HUKM reported cases July 2005 - June 2006 in Malaysia

Thompson and Kurzrock (2004) in an extensive review mentioned that $90 \%$ of the world population carried EBV and pointed out that variation in different countries might be due to their environmental, geographical, socioeconomic and hygienic conditions.

The results of the present study are similar to those of Giovanni et al. (2001) who reported in Papua New Guinea that 93\% people in rural area and 100\% in city less than 25 years of old were positive for EBV antibodies.

It is evident from different studies that about $100 \%$ of older people carry EBV, which make them vulnerable to diseases if immunosuppression occurs because of AIDS, organ transplantation or genetic immune disorders.

In the present study, EBV-specific IgG and IgM antibodies were determined. It was found (Figs. 1, 2, 3 and 4) that the percentage of samples positive to IgM was less than for IgG. IgM is the antibody detected early during any infection and very few patients report to the hospital at the early stage of an illness. Therefore detection of EBV specific IgM in a lower percentage of cases is not unusual. In most cases in the present study IgM-positive cases were also positive to IgG.

An attempt was taken to determine the epidemiological pattern of EBV infection in different months. It was observed (Fig. 4) that the variation was not significant.

It might be due to the fact that in Malaysia environmental temperature varies little round the year.

The present study showed that as in other counties, a high percentage of Malaysian people carry EBV-specific antibodies. The carrier population should remain aware of any immunosuppression with HIV and other diseases that may 
reactivate EBV. In addition EBV carriers should remain cautious so that spreading EBV does not occur through their saliva. Saliva is a rich source of virus through which it disseminates easily and this can shed for years after initial infection (Gerber et el., 1972). Rinaldo (1990) expressed the opinion that herpesvirinae cause immunosuppression by inhibition of cytokine production and direct interaction of virus with Major Histocompatibility Complex components, which have clinical significance.

\section{References}

Anon 2006a: Clinical utility of EBV antibody screening to identify individual susceptible to nasopharyngeal-carcinoma. www.clinicaltrials.gov/ct/gui/show

Anon 2006b: Enzygnost ${ }^{\circledR}$ Anti EBV/IgG, Enzygnost ${ }^{\circledR}$ Anti-EBV/IgM. Dade Bearing Marburg $\mathrm{GmbH}$, Emil-von-Behring-Str-76, D-35041, Marburg, Germany pp. 1-76.

Gerber P, Lucas S, Nonoyama M, Lucas S, Perlin E, Goldstein LI 1972: Oral excretion of Epstein-Barr viruses by healthy subjects and patients with infectious mononucleosis. Lancet 2 988-989.

Giovanni R., Robert T D, Theresa M W, Loredana S L, Owen P M, Massimo A, Barbara S, Barbara E, Edward P 2001: Human Herpesvirus-8 and Other Viral Infections. Papua New Guinea Center for Disease Control and Prevention 75.

Haque T, Iliadou P, Hossain A, Crawford DH 2001: Sero-epidemiological study of Epstein-Barr virus infection in Bangladesh. Journal of Medical Virology 48 17-21.

Takeuchi K, Tanaka-Taya K, Kazuyama Y, Ito YM, Hashimoto S, Fukayama M, Mori S 2006: Prevalence of Epstein-Barr virus in Japan: Trends and future prediction. Pathology International 56 112-118.

Thompson MP, Kurzrock R 2004: Epstein-Barr virus and cancer: Clinical Cancer Research 10 803-821.

Rinaldo CR 1990: Immunosuppression by herpes viruses. Annual Review of Medicine $4 \mathbf{1}$ 331-338. 